Research Article

\title{
Does Timing of Antenatal Care Initiation and the Contents of Care Have Effect on Caesarean Delivery in Ethiopia? Findings from Demographic and Health Survey
}

\author{
Getnet Gedefaw $\mathbb{D}^{1},{ }^{1}$ Fikadu Waltengus, ${ }^{2}$ and Asmamaw Demis $\mathbb{i D}^{3}$ \\ ${ }^{1}$ School of Midwifery, College of Health Sciences, Woldia University, P.O.Box: 400, Woldia, Ethiopia \\ ${ }^{2}$ Department of Midwifery, College of Medicine and Health Sciences, School of Health Science, Bahir Dar University, \\ Bahir Dar, Ethiopia \\ ${ }^{3}$ School of Nursing, College of Health Sciences, Woldia University, P.O.Box: 400, Woldia, Ethiopia \\ Correspondence should be addressed to Getnet Gedefaw; gedefawget@gmail.com
}

Received 10 April 2021; Accepted 3 August 2021; Published 10 August 2021

Academic Editor: Animesh Jain

Copyright (c) 2021 Getnet Gedefaw et al. This is an open access article distributed under the Creative Commons Attribution License, which permits unrestricted use, distribution, and reproduction in any medium, provided the original work is properly cited.

\begin{abstract}
Background. Antenatal care (ANC) is an important preventive set of core healthcare services through pregnancy. Caesarean deliveries are significantly increasing in many low-, middle-, and high-income countries. However, overuse of the caesarean section service interferes with the quality and cost of the procedure. Hence, this study aimed to assess the effect of timing of first antenatal care initiation and the contents of care on caesarean delivery. Methods. A population level cross-sectional study was conducted with a total of 4757 study participants. The multivariable analysis was computed using the setup of 3 models. Results. The rate of caesarean section among women who initiated antenatal care in the first trimester was $1.32 \%(95 \% \mathrm{CI}=0.91-4.21)$. Women initiated antenatal care in the first trimester $(\mathrm{AOR}=2.74 ; 95 \% \mathrm{CI}=1.49-6.2)$ and received contents of care $(\mathrm{AOR}=1.98$; $95 \% \mathrm{CI}=1.24-3.78]$ )were more likely to have caesarean section delivery as compared to their counterparts. Conclusion. Caesarean section among women who initiated ANC in the first trimester is low. The finding suggests ANC initiated early (within 16 weeks) can have a positive impact on caesarean section delivery. In addition, being urban residents, primipara women, initiating antenatal care before 16 weeks, received contents of care, and having antenatal care visits three and more increase the odds of having caesarean section. As a result, different obstetric, medical, and surgical complications are detected and managed as early as possible.
\end{abstract}

\section{Introduction}

Caesarean delivery refers to the delivery of the fetus through a surgical incision made through the abdominal wall (laparotomy) and the uterine wall (hysterotomy). According to the World Health Organization (WHO), caesarean deliveries are the most common surgical procedures in the world, nearly 18 million are performed yearly worldwide [1]. Although caesarean delivery has played a significant role in lowering both unfavorable maternal and fetal outcomes during the past decade, however, caesarean delivery has been associated with a variety of short- and long-term maternal and neonatal complications [2]. Although the rate of caesarean section is significantly increasing, however, it is not associated with improved outcomes for mothers and newborns and deliveries may not lead to better health outcomes for women or neonates who do not require the procedure [3-6].

The provision and uptake of quality and timely antenatal care (ANC) are an essential element of efforts to improve health outcomes for women and newborn babies. Antenatal consultations assist in the early identification and treatment of complications during pregnancy. Women not initiating ANC early may lead to late diagnosis of complications which might have the potential to detrimentally affect maternal and fetus health. As a result, it contributes to maternal mortality, 
premature labor, and preterm babies and intrauterine deaths are significantly increasing. In addition, late initiation of antenatal care might be responsible for complications such as preeclampsia, anemia, and low birth weight among teenage and unmarried women [7].

Studies showed that maternal age, level of education, wealth, parity, and age at marriage were statistically significantly associated with the timing of initiation of antenatal care attendance [8]. Different studies evidenced that women who started ANC attendance early, received components of care, and attended frequently were more likely to be assisted during childbirth by a skilled attendance compared to those who initiated ANC late and attended only a few visits. When the number of receiving contents of antenatal care visits increases, the woman is expected to escalate women's perception about birth preparedness and complication readiness besides other contents of antenatal care [9].

Early initiation of antenatal care helps health workers to provide timely information and services according to the gestational age and the health condition of both the mother and the fetus. But, mothers who attend antenatal care late miss the opportunity to receive health information and interventions such as early detection of HIV, malaria, and anemia prophylaxis and prevention or management of complications [10].

According to the World Health Organization (WHO), every pregnant woman in developing countries should seek ANC within the first trimester of gestation. While preparing for safe childbirth is an essential part of ANC, the timely initiation of the first ANC visit is an important element. Studies have shown that early ANC attendance (during the first trimester of pregnancy) plays a major role in the early detection and treatment of maternal health problems in pregnancy and serves as a good basis for proper management during and after childbirth [11].

Ethiopia has developed different strategies, programs, and policies to improve maternal health services utilization. Caesarean section deliveries, facility delivery cares, family planning services, antenatal care services, and postnatal care services are provided free of charge [12-15].

Early initiation of antenatal care is more expedient in the role of early detection and management of problems related to pregnancy and preventing adverse complications during pregnancy, although women are still starting antenatal care visits in their late pregnancy $[16,17]$. Despite antenatal care reduces maternal and perinatal morbidity and mortality with the help of early detection and treatment of pregnancy-related complications, still the contents of care given for women who attended antenatal care are still low. The likelihood of achieving skilled birth attendant, antenatal care, and institutional delivery is less likely to achieve the national target of reproductive health services. Assessing factors and barriers that affect caesarean delivery are crucial to achieving the national target. More studies, with large sample sizes, are needed to identify the factors associated with caesarean delivery in Ethiopia. Therefore, this study tried to examine the effect of timing of antenatal care visits (initiated antenatal care in the first trimester) and the component of antenatal care services on caesarean section delivery.

\section{Materials and Methods}

2.1. Study Area and Design. This research analyzed factors reported by the 2016 Ethiopia Demographic and Health Survey (EDHS) from the fourth version of the survey. We used the 2016 EDHS data, the fourth DHS in Ethiopia, which was collected from January 18 to June 27, 2016, for this analysis. It is a community-based, nationally representative, cross-sectional data collected from all 9 regions and two city administrations [18].

2.2. Data and Sampling Procedures. Data for this study were retrieved from the 2016 EDHS, which used a weighted multistage, stratified cluster sampling approach. The 2016 EDHS data employed a two-stage stratified cluster sampling procedure for nine regional states and two city administrations. A total of 645 enumeration areas (202 in urban areas and 443 in rural areas) were selected using a probability proportional to the enumeration size. All women aged 15-49 who were either permanent residents of the selected households or visitors who stayed in the household the night before the survey were eligible to be interviewed for interviewer-administered structured questionnaires. A total of 15,683 women aged 15-49 years were interviewed in the 2016 EDHS, of which 7,590 women had at least one live birth in the last 5 years before the survey [18]. Two thousand eight hundred and thirty three women were excluded from the study as 2818 women did not attend antenatal care at all and 15 women did not know to either attend their antenatal care follow up or not. Overall, the included sample size for this study was 4757 women who had at least one antenatal care during the recent pregnancy.

\subsection{Measurement of Outcomes}

2.3.1. Caesarean Delivery. Women who responded as live births delivered by caesarean section coded as yes " 1 " and no "0."

\subsection{Exposure Measurement}

2.4.1. Covariates. Socioeconomic and sociodemographic antenatal care service-related factors, fertility, and reproductive health-related characteristics were included as covariates.

Socioeconomic and sociodemographic characteristics: region, residence, age of the mother, educational status of the mother, educational status of the husband, religion, marital status, working status of the mother, birth order of the recent child, and wealth index.

Antenatal care service-related factors: timing of first antenatal care, frequency of antenatal care visit, and content of antenatal care.

Fertility and reproductive health-related factors: parity, the status of pregnancy (whether wanted or not), age at 
first birth, number of living children, and birth weight of the recent pregnancy.

2.5. Data Processing and Analysis. Data were analyzed using SPSS version 20 statistical software. Complex sample survey (stratified/clustered) sampling designs were used to correctly calculate unequal probabilities of selection with weighted data. Rao-Scott chi-square was used to examine the bivariate associations between each covariate and caesarean delivery via adjusting complex survey sampling. Weighting was used to correct for nonresponse and disproportionate sampling. Both bivariate and multivariable logistic regression analyses were performed to identify the impact of timing of antenatal care and content of care on caesarean delivery. Both descriptive and analytical statistics were calculated. Covariates in the bivariate logistic regression with a $p$ value of less than 0.25 were a candidate to be fitted into the multivariable logistic regression to control the possible effects of confounders and assess the association between caesarean section and timing of antenatal care initiation and contents of care using the control model. In the multivariable analysis, variables with a $p$ value $<0.05$ were declared as statistically significant with odds ratios with their corresponding $95 \%$ confidence intervals (CI). Multicollinearity was checked using the standard error (SE) with a cut-off point of below 2. As a result, we declared that no multicollinearity was existed in this study [18]. Moreover, the association between the main independent variables (timing of antenatal care and received contents of care) with caesarean section delivery was investigated by using binary logistic regression (model 1).

In model 2, variables that demonstrated a significant association (with a $p$ value of $\leq 0.05$ ) in bivariate analysis excluding timing of antenatal care and received contents of care were exported to a multiple logistic regression model for multivariate analysis. Finally, the timing of antenatal care, parity, number of antenatal care visit, and residence were significantly associated with caesarean section deliveries in the final model after controlling all the possible confounders (model 3).

\subsection{Operational Definition}

Timely initiation of antenatal care: women who receivied antenatal care from a skilled provider for the most recent birth within 3 months of her pregnancy.

Caesarean section: a surgical procedure involving incision of the walls of the abdomen and uterus for delivery of offspring.

Content of antenatal care: according to WHO recommendation, women should receive the following core set maternal services: measuring blood pressure, a urine sample taken, a blood sample taken, told about pregnancy complications, tetanus toxoid vaccination, taken drugs for intestinal parasites, and iron tablets/ syrup supplementation.
2.7. Ethical Approval and Consent to Participate. All the available datasets were obtained from the DHS website (https://dhsprogram.com/); through registering with the DHS website, no ethical approval was required. Institutional Review Board of Woldia University waived the requirement for informed consent for EDHS data. All the authors have no special access privileges to this dataset. To access and get the data, authors should register and log in. While we request the title of the project, the co-author's name and e-mail address and a brief description of the study should be clearly stated. Then, the researchers continue to select the country, dataset, and year of the survey. Within a few days after requesting, the demographic and health survey team will get permission to download the dataset via e-mail of the corresponding author. After permission is stated, the author can $\log$ in and select the specific data with the important format the author wants [18].

\section{Results}

3.1. Socioeconomic and Sociodemographic Characteristics. Out of the 4757 study participants, more than two-thirds of the mothers were rural residents. Almost all (93.7\%) were married or in union. More than half (69\%) were orthodox Christians in terms of religion. Regarding the level of education, most of the mothers (54\%) did not attend formal education. Given the wealth index, $22.5 \%$ of the mothers were in the richest wealth quintile level (Table 1).

\subsection{Contents of Antenatal Care, and Its Relation with Cae-} sarean Section Deliveries. In this survey, all women who initiated antenatal care follow-up (in the first trimester) were getting routine components of antenatal care services such as blood pressure measurement, urine analysis, blood group and $\mathrm{Rh}$ determination, prophylaxis of iron supplementation, and tetanus toxoid vaccination as compared to women who initiated antenatal care services after 16 weeks of gestation. In this study, 2654, pregnant women, were received antenatal care visits in the first trimester of pregnancy in the health facility. Around twenty-two and twenty-one percent of the population size received tetanus toxoid vaccination and their blood pressure was measured, respectively (Table 2).

3.3. Obstetrics, Fertility, and Reproductive Health-Related Characteristics. Out of the overall study participants, nearly half of the $2342(49.2 \%)$ pregnant women had initiating antenatal care visits within 16 weeks of pregnancy (early). More than two-thirds of the pregnancy was wanted and then followed by wanted later (Table 3).

After controlling confounders in each model $(1,2$, and 3 ), the timing of antenatal care, parity, contents of antenatal care, number of antenatal care visits, and residence were the factors significantly associated with caesarean section deliveries in the final model.

Women who initiated their antenatal care visit early (within 16 weeks) of pregnancy (AOR $=2.74 ; 95 \%$ $\mathrm{CI}=1.49-6.2)$ were nearly three times more likely to 
TABLE 1: Socioeconomic and sociodemographic characteristics $(N=4757)$.

\begin{tabular}{|c|c|c|}
\hline Variable & Frequency & Percent \\
\hline \multicolumn{3}{|l|}{ Region } \\
\hline Tigray & 481 & 10.1 \\
\hline Afar & 36 & 0.7 \\
\hline Amhara & 1102 & 23.2 \\
\hline Oromia & 1605 & 33.8 \\
\hline Somali & 117 & 2.4 \\
\hline Benishangul & 56 & 1.1 \\
\hline SNNPR & 1111 & 23.5 \\
\hline Gambela & 15 & 0.3 \\
\hline Harari & 13 & 0.26 \\
\hline Addis Ababa & 192 & 4.04 \\
\hline Dire Dawa & 29 & 0.6 \\
\hline \multicolumn{3}{|l|}{ Religion } \\
\hline Orthodox & 2024 & 42.5 \\
\hline Muslim & 1568 & 33 \\
\hline $\begin{array}{l}\text { Others (protestant, Catholic, and } \\
\text { traditional) }\end{array}$ & 1165 & 24.5 \\
\hline \multicolumn{3}{|l|}{ Educational status of the mother } \\
\hline No education & 2569 & 54 \\
\hline Primary & 1574 & 33.1 \\
\hline Secondary and above & 614 & 12.9 \\
\hline \multicolumn{3}{|l|}{ Residence } \\
\hline Urban & 870 & 18.29 \\
\hline Rural & 3887 & 81.71 \\
\hline \multicolumn{3}{|l|}{ Marital status } \\
\hline Married/in union & 4470 & 93.7 \\
\hline Others & 287 & 6.3 \\
\hline \multicolumn{3}{|l|}{ Birth order } \\
\hline First & 1119 & 23.5 \\
\hline Second to fourth & 2083 & 43.8 \\
\hline Five or more & 1555 & 32.7 \\
\hline \multicolumn{3}{|l|}{ Maternal age } \\
\hline$\leq 19$ & 749 & 15.8 \\
\hline $20-34$ & 3418 & 71.8 \\
\hline$\geq 35$ & 590 & 12.4 \\
\hline \multicolumn{3}{|l|}{ Mothers' current working status* } \\
\hline Unemployed & 1507 & 31.7 \\
\hline Employed & 3250 & 68.3 \\
\hline \multicolumn{3}{|l|}{ Wealth index } \\
\hline Poorest & 791 & 16.6 \\
\hline Poorer & 935 & 19.7 \\
\hline Middle & 993 & 20.8 \\
\hline Richer & 966 & 20.4 \\
\hline Richest & 1072 & 22.5 \\
\hline \multicolumn{3}{|l|}{ Educational level of the husband } \\
\hline No education & 1810 & 38 \\
\hline Primary & 1813 & 38.2 \\
\hline Secondary and above & 846 & 17.8 \\
\hline
\end{tabular}

undergo caesarean section deliveries than the women who initiate their antenatal care follow-up after three months of pregnancy.

The odds of having caesarean deliveries $(\mathrm{AOR}=2.13$; $95 \% \mathrm{CI}=1.5-3.72$ ) were two times higher among primipara women than multipara women.
TABLE 2: Percentage of women receiving components/contents of antenatal care $(N=4757)$.

\begin{tabular}{lccc}
\hline Variable & Frequency & Percent & Odds ratio \\
\hline Blood pressure taken & & & \\
Yes & 1175 & 24.7 & $21.26(14.09-21.06)$ \\
No & 3582 & 75.3 & \\
\hline Blood sample taken & & & \\
Yes & 1303 & 27.4 & $20.5(16.6-24.4)$ \\
No & 3454 & 72.6 & \\
\hline Urine sample taken & & & \\
Yes & 3147 & 66.2 & $18.68(15.1-22.27)$ \\
No & 1610 & 33.8 & \\
\hline Told about pregnancy & complication \\
Yes & 2142 & 45 & \\
No & 2615 & 55 & $17.12(10.17-15.26)$ \\
\hline Iron tablets/syrup supplementation & & \\
Yes & 2855 & 60 & \\
No & 1889 & 39.7 & $17.58(14.09-21.06)$ \\
Do not know & 13 & 0.3 & \\
\hline Tetanus toxoid vaccination & & \\
Received injection & 3466 & 72.9 & \\
Not received & 1149 & 24.2 & $22.26(17.9-26.62)$ \\
Do not know & 142 & 2.9 & \\
\hline Taken drugs for intestinal parasites & & \\
Yes & 364 & 7.65 & \\
No & 4311 & 90.6 & $6.02(4.04-8.0)$ \\
Do not know & 82 & 1.75 & \\
\hline \multicolumn{4}{l}{} \\
\hline
\end{tabular}

Similarly, the chance of having caesarean section deliveries $(\mathrm{AOR}=4.78 ; 95 \% \mathrm{CI}=2.8-8.75)$ were nearly four times among urban residents compared to people living in rural areas.

Women having antenatal care visits more than four times $(\mathrm{AOR}=2.85 ; 95 \% \mathrm{CI}=1.38-5.73)$ were more likely to have caesarean section deliveries compared to women who had less than and equal to four antenatal visits.

Women who received any contents of antenatal care were $(\mathrm{AOR}=1.98 ; 95 \% \mathrm{CI}=1.24-3.78)$ times more likely to have caesarean section as compared to their counterparts (Table 4).

\section{Discussion}

This study result revealed that among women who attended antenatal care visits in the first trimester, around three in a hundred women had caesarean delivery. The rate of caesarean delivery service use among women who initiated antenatal care visits within 16 weeks of pregnancy was $(1.32 \%(95 \% \mathrm{CI}=0.91-4.21))$ which is low as compared to the WHO caesarean section recommendation rate (5-15\%).

The rate of caesarean section is lower than the study conducted in different regions of Ethiopia, Addis Ababa [19], Yirga Alem [20], Hawassa [21], Finote Selam [22], and Chiro [23], and Saudi Arabia [24] and Jordan [25]. The possible justification for lowering of caesarean section is the difference of the study population. i.e., considering study participants who were only women who had attended and received contents of antenatal care in the first trimester 
TABLE 3: Obstetrics, fertility, and reproductive health-related characteristics $(N=4757)$.

\begin{tabular}{lcc}
\hline Variable & Frequency & Percent \\
\hline Timing of first antenatal care & & \\
Early (within 16 weeks of pregnancy) & 2342 & 49.2 \\
Late (above 16 weeks of gestation) & 2415 & 50.8 \\
\hline Wanted status of a child ${ }^{*}$ & & \\
Wanted then & 3612 & 75.9 \\
Wanted later & 821 & 17.3 \\
Wanted no more & 324 & 0.68 \\
\hline Parity & & \\
Multipara** & 3638 & 76.5 \\
Primipara & 1119 & 23.5 \\
\hline Age at first birth & & \\
$\leq 19$ & 2919 & 61.4 \\
$20-34$ & 1828 & 38.4 \\
$\geq 35$ & 10 & 0.2 \\
\hline Number of children & & \\
$1-2$ & 1960 & 41.2 \\
$3-5$ & 1727 & 36.3 \\
6 and more & 1070 & 22.5 \\
\hline Number of antenatal care visits & \\
$\leq 4$ & 3536 & 74.33 \\
$>4$ & 1221 & 25.66 \\
\hline Birth weight of the recent pregnancy & & \\
$\leq 2499$ gram & 259 & 5.4 \\
$2500-3999$ gram & 797 & 16.8 \\
$\geq 4000$ gram & 3701 & 77.8 \\
\hline
\end{tabular}

${ }^{*}$ Percent distribution of births to women aged 15-49 in the 5 years preceding the survey, including current pregnancies, by planning status of the birth wanted then, wanted later, or not wanted at all. ${ }^{* *}$ A woman that has been having had more than one pregnancy resulting in viable offspring.

of pregnancy might reduce the magnitude of caesarean section since this study focused on the impact of early initiation of antenatal care on caesarean delivery. Furthermore, this study showed evidence of women having early initiation of antenatal care and received contents of standared care with qualified professionals have a impact on mode of delivery.

Early initiation of antenatal care has a significant impact on caesarean section delivery preference. This study finding is supported and showed the overall antenatal care was positively associated with caesarean delivery in Jordan [25] and Addis Ababa [26]. Antenatal care is an indicator for timing, number of antenatal visits, and content of services received during antenatal care visits. Women who received adequate antenatal care early or within three months of pregnancy were more likely to have caesarean section delivery compared to those women who initiated their first antenatal care after 16 weeks of pregnancy.

Keeping in view the significance of antenatal care utilization in determining the mode of delivery, the WHO recommends every pregnant woman to have at least four antenatal care visits during her pregnancy. Mothers who received antenatal care more than four times delivered babies by caesarean section. Although the reason for this is still unclear, the risk-avoiding behavior of gynecologists towards women who reported having pregnancy complications could be one of the reasons for this preference for caesarean delivery. Besides the effect of prenatal education on antenatal clients, early initiation of antenatal care is aimed to prevent health risks, early detection of abnormalities, and institution of corrective measures if possible and preparation of both the woman and fetus and to ensure a good start of life for each newborn child [27, 28].

Pregnant women require knowledge on caesarean delivery. The antenatal visit is the right moment to provide counseling related to this mode of delivery. Inadequate antenatal care in visits could have influenced the caesarean delivery because antenatal care is the opportunity to anticipate macrosomia and induce labor before the expected delivery date and avoid excessive fetal growth [29].

In this study, nearly half of the ANC attended women $49.2 \%$ were initiating their antenatal care follow-up within three months of pregnancy. This study finding is higher than the study conducted in Tigray [30] (27.5\%) and in the global estimate [31] 24\%. This might be because; this study is a national survey population-level analysis which yields a significant representative than a single study. Besides, this inconsistency could be attributed to the scope of the study, the fact that EDHS covered more remote.

Women who resided in urban areas were more than three times likely to have caesarean section deliveries compared to women who resided in rural areas. This factor was also significantly associated with CS delivery in studies conducted in Ethiopia, Mizan Aman [32]. Although women who resided in rural areas may initiate antenatal care, unfortunately, they withdraw their follow-up because of poor counseling on timing, number, and contents of the antenatal care follow-up. As a result, they have no more knowledge on birth preparedness, complication readiness, and mode of delivery as well as where they have to go if they feel labor pain in their home. On the contrary, women who live in urban areas have a chance to access the health facility with good adherence to prenatal counseling on danger signs and they fear labor pain since epidural analgesia during labor is not practiced in our country; therefore, most of the women preferred to have caesarean section deliveries. Even women who are living in urban areas have a high chance of going to private hospitals and caesarean section might be done without being medically indicated with maternal preference.

Caesarean section deliveries are more frequent amongst primipara women. This study finding is supported by the studies conducted in Jordan [25], Vietnam [33], India [34], and Bangladesh [35]. Increasing parity was found to be associated with decreased odds of caesarean section delivery which is consistent with the clinical practice in our country. Nulliparity is associated with a higher rate of induction of labor, cephalopelvic disproportion; as a result, caesarean section delivery is anticipated [36]. Women who received contents of antenatal care were more likely to give birth through caesarean section delivery as compared to their counterparts, since initiating early antenatal care checkups could increase the strength of the relationship between ANC and reductions in neonatal and maternal mortality through received contents of antenatal care [8]. 
TABLE 4: Bivariate and multivariable logistic regression

\begin{tabular}{|c|c|c|c|c|c|}
\hline \multirow{2}{*}{ Variable } & \multicolumn{2}{|c|}{ Caesarean delivery } & \multirow{2}{*}{$\begin{array}{c}\text { Model } 1 \\
\text { COR }(95 \% \text { CI })\end{array}$} & \multirow{2}{*}{$\begin{array}{c}\text { Model } 2 \\
\text { AOR }(95 \% \text { CI })\end{array}$} & \multirow{2}{*}{$\begin{array}{c}\text { Model } 3 \\
\text { AOR (95\% CI) }\end{array}$} \\
\hline & No & Yes & & & \\
\hline \multicolumn{6}{|c|}{ Timing of antenatal care } \\
\hline Early & $2311(48.6 \%)$ & $31(0.7 \%)$ & $4.56(2.5-8.2)$ & & $2.74(1.49-6.2)^{*}$ \\
\hline Late & $2277(47.7 \%)$ & $138(2.9 \%)$ & 1 & & 1 \\
\hline \multicolumn{6}{|c|}{ Received contents of care } \\
\hline Yes & 2029 & 113 & $2.54(1.6-3.97)$ & & $1.98(1.24-3.78)^{*}$ \\
\hline No & 2559 & 56 & 1 & & 1 \\
\hline \multicolumn{6}{|l|}{ Birth order } \\
\hline One & $1048(22 \%)$ & $70(1.5 \%)$ & & $6.43(2.8-11.8)$ & $4.33(0.72-8.6)$ \\
\hline Two to four & $2000(42 \%)$ & $84(1.8 \%)$ & & $3.2(1.87-7.32)$ & $2.97(0.6-5.2)$ \\
\hline Five and more & $1540(32.4 \%)$ & $15(0.3 \%)$ & & 1 & 1 \\
\hline \multicolumn{6}{|l|}{ Parity } \\
\hline Primipara & $1048(22 \%)$ & $71(1.5 \%)$ & & $2.43(1.76-3.92)$ & $2.13(1.5-3.72)^{*}$ \\
\hline Multipara & $3540(74.4 \%)$ & $98(2.1 \%)$ & & 1 & 1 \\
\hline \multicolumn{6}{|c|}{ Educational status of the mother } \\
\hline No education & $2532(53.2 \%)$ & $37(0.8 \%)$ & & 1 & 1 \\
\hline Primary & $1519(31.9 \%)$ & $55(1.2 \%)$ & & $2.54(1.34-4.67)$ & $0.98(0.67-3.7)$ \\
\hline Secondary and above & $537(11.3 \%)$ & $77(1.6 \%)$ & & $9.79(5.43-17.65)$ & $1.25(0.47-2.74)$ \\
\hline \multicolumn{6}{|l|}{ Wealth index } \\
\hline Poorer & $924(19.4 \%)$ & $12(0.2 \%)$ & & $0.88(0.25-3.16)$ & $0.91(0.62-3.45)$ \\
\hline Poorest & $780(16.4 \%)$ & $11(0.2 \%)$ & & 1 & 1 \\
\hline Middle & $977(20.5 \%)$ & $16(0.3 \%)$ & & $1.15(0.4-3.38)$ & $1.34(0.67-4.8)$ \\
\hline Richer & $950(20 \%)$ & $15(0.3 \%)$ & & $1.12(0.36-3.43)$ & $0.75(0.12-1.94)$ \\
\hline Richest & $957(20.1 \%)$ & $15(2.4 \%)$ & & $8.41(3.54-19.98)$ & $1.54(0.85-3.87)$ \\
\hline \multicolumn{6}{|l|}{ Residence } \\
\hline Rural & $3830(80.5 \%)$ & $57(1.2 \%)$ & & 1 & 1 \\
\hline Urban & $758(15.9 \%)$ & $112(2.3 \%)$ & & $7.21(4.7-10.23)$ & $4.78(2.8-8.75)^{*}$ \\
\hline \multicolumn{6}{|c|}{ Number of antenatal care visits } \\
\hline$\leq 4$ visits & $3433(72.17 \%)$ & $103(2.17 \%)$ & & 1 & 1 \\
\hline$>4$ visits & $1023(21.5 \%)$ & $198(4.16 \%)$ & & $6.45(2.5-9.2)$ & $2.85(1.38-5.73)^{*}$ \\
\hline
\end{tabular}

${ }^{*}$ The variable is significant with a $p$ value of less than 0.05 .

\section{Conclusion}

Caesarean section among women who initiated ANC in the first trimester is low. The finding suggests ANC initiated early (within 16 weeks) can have a positive impact on caesarean section delivery. In addition, being urban residents, primipara women received contents of care, initiating antenatal care before 16 weeks, and having antenatal care visits three and more increase the odds of having caesarean section in Ethiopia. Initiating the antenatal care early can increase the uptake of all contents of antenatal care and enhances the investigation of women further for different obstetric, medical, and surgical complications as early as possible. Avoiding wrong perceptions about the timing of ANC is related to the lack of knowledge about the benefit of early antenatal care and cultural and traditional beliefs related to health care-seeking practices during pregnancy. As a result, more efforts should be taken to increase the knowledge of mothers about the early initiation of antenatal care.

5.1. Limitation. The temporal cause and effect relationship might not be possible due to the natural effect of crosssectional study design.

\section{Abbreviations}

ANC: Antenatal care

CI: Confidence interval

CS: Caesarean section

IBF: Initiate breastfeeding

OR: $\quad$ Odds ratio

UNICEF: United Nations International Children's Emergency Fund

WHO: World Health Organization.

\section{Data Availability}

All the organized datasets that support the finding of the study are available upon request from the corresponding author.

\section{Conflicts of Interest}

All authors declare that they have no conflicts of interest.

\section{Authors' Contributions}

GG and $\mathrm{AD}$ were involved in the design, selection of variables, and statistical analysis. GG, AD, and FW were 
involved in manuscript writing. All authors read and approved the final draft of the manuscript.

\section{Acknowledgments}

The authors are indebted to the DHS Program for providing them permission to use the 2016 EDHS data for this analysis.

\section{References}

[1] D. Lyell, M. Power, K. Murtough et al., "Surgical techniques at cesarean delivery: a U.S. Survey," Surgery Journal, vol. 2, no. 4, pp. e119-e125, 2016.

[2] W. F. Rayburn and A. L. Strunk, "Profiles about practice settings of American college of obstetricians and gynecologists fellows," Obstetrics \& Gynecology, vol. 122, no. 6, pp. 1295-1298, 2013.

[3] V. Briand, A. Dumont, M. Abrahamowicz, M. Traore, L. Watier, and P. Fournier, "Individual and institutional determinants of caesarean section in referral hospitals in Senegal and Mali: a cross-sectional epidemiological survey," BMC Pregnancy and Childbirth, vol. 12, p. 114, 2012.

[4] M. J. Hyde, A. Mostyn, N. Modi, and P. R. Kemp, "The health implications of birth by caesarean section," Biological Reviews, vol. 87, no. 1, pp. 229-243, 2012.

[5] J. Villar, G. Carroli, N. Zavaleta et al., "Maternal and neonatal individual risks and benefits associated with caesarean delivery: multicentre prospective study," BMJ, vol. 335, no. 7628, p. 1025, 2007.

[6] A. P. Betrán, J. Ye, A.-B. Moller, J. Zhang, A. M. Gülmezoglu, and M. R. Torloni, "The increasing trend in caesarean section rates: global, regional and national estimates: 1990-2014," PLoS One, vol. 11, no. 2, Article ID e0148343, 2016.

[7] Y. R. Paudel, T. Jha, and S. Mehata, "Timing of first antenatal care (ANC) and inequalities in early initiation of ANC in Nepal," Frontiers in public health, vol. 5, p. 242, 2017.

[8] S. Agha and H. Tappis, "The timing of antenatal care initiation and the content of care in Sindh, Pakistan," BMC Pregnancy and Childbirth, vol. 16, no. 1, p. 190, 2016.

[9] K. Gross, S. Alba, T. R. Glass, J. A. Schellenberg, and B. Obrist, "Timing of antenatal care for adolescent and adult pregnant women in south-eastern Tanzania," BMC Pregnancy and Childbirth, vol. 12, no. 1, p. 16, 2012.

[10] T. Belayneh, M. Adefris, and G. Andargie, "Previous early antenatal service utilization improves timely booking: crosssectional study at university of Gondar hospital, northwest Ethiopia," Journal of pregnancy, vol. 2014, Article ID 132494, 7 pages, 2014

[11] A. K. Manyeh, A. Amu, J. Williams, and M. Gyapong, "Factors associated with the timing of antenatal clinic attendance among first-time mothers in rural southern Ghana," $B M C$ Pregnancy and Childbirth, vol. 20, no. 1, p. 47, 2020.

[12] H. Zelelew, "Health care financing reform in Ethiopia: improving quality and equity," Health Systems, vol. 20, p. 20, 2018.

[13] Federal democratic republic of Ethiopia, National Reproductive Health Strategy, Ministry of health, Addis Ababa, Ethiopia, 2014.

[14] H. Banteyerga, "Ethiopia's health extension program: improving health through community involvement," MEDICC review, vol. 13, no. 3, pp. 46-49, 2011.

[15] A. M. Karim, K. Admassu, J. Schellenberg et al., "Effect of Ethiopia's health extension program on maternal and newborn health care practices in 101 rural districts: a doseresponse study," PLoS One, vol. 8, no. 6, Article ID e65160, 2013.

[16] T. Gudissa Damme, D. Workineh, and A. Gmariam, "Time of antenatal care booking and associated factors among pregnant women attending ambo town health facilities, Central Ethiopia," Journal of Gynecology and Obstetrics, vol. 3, no. 5, pp. 103-106, 2015.

[17] W. Lerebo and A. Kidanu, "Magnitude and associated factors of late booking for antenatal care in public health centers of Adigrat town, Tigray, Ethiopia," Clinics in Mother and Child Health, vol. 12, p. 171, 2015.

[18] G. Gedefaw, M. H. Goedert, E. Abebe, and A. Demis, "Effect of cesarean section on initiation of breast feeding: findings from 2016 Ethiopian Demographic and Health Survey," PLoS One, vol. 15, no. 12, Article ID e0244229, 2020.

[19] A. Bizuneh and G. Ayana, "Indications and outcomes of emergency caesarean section at St Paul's HospitalMedical College, Addis Ababa, Ethiopia 2017: (afoul month retrospective cohort study)," Invest Gynecol Res Women's Health.vol. 2, no. 2, 2017.

[20] T. Tesfaye, D. Hailu, N. Mekonnen et al., "Magnitude of maternal complication and associated factors among mothers undergone cesarean section at Yirgalem general hospital, SNNPR, Ethiopia," International Journal of Health Sciences \& Research, vol. 7, no. 5, pp. 264-272, 2017.

[21] Z. Tenaw, Z. Y Kassa, G Kassahun, and A Ayenew, "Maternal preference, mode of delivery and associated factors among women who gave birth at public and private hospitals in Hawassa city, southern Ethiopia," Annals of Global Health, vol. 85, no. 1, pp. 1-7, 2019.

[22] K. Melaku, "Cesarean section rate, maternal and fetal outcome of birth following cesarean section at Finoteselam hospital, northwest Ethiopia: a descriptive retrospective data," Global Journal of Medical Research: Gynecology \& Obstetrics, vol. 16, no. 3, 2016.

[23] MeleseA, Magnitude Of Cesarean Section Delivery And Its Associatedfactors Among Mothers Who Gave Birth At Public Hospitalsin North Wollo Zone, Northern Ethiopia, Haramaya University, Dire Dawa, Ethiopia, 2019.

[24] H. ZakaiGhadeer, "Prevalence of caesarean section and its indicating factors among pregnant women attending delivery at king abdulaziz university hospital, jeddah city during 2016," EC Gynaecology, vol. 2, pp. 43-51, 2018.

[25] A. M. Batieha, S. A. Al-Daradkah, Y. S. Khader, A. Basha, F. Sabet, and T. Z. Athamneh, "Cesarean section: incidence, causes, associated factors and outcomes: a national prospective study from Jordan," GynecolObstet Case Report, vol. 3, no. 3, p. 55, 2017.

[26] Y. T. Bayou, Y. J. Mashalla, and G. Thupayagale-Tshweneagae, "Patterns of caesarean-section delivery in Addis Ababa, Ethiopia," African journal of primary health care \& family medicine, vol. 8, no. 2, pp. e1-e6, 2016.

[27] World Health Organization, Provision of Effective Antenatal Care: Integrated Management of Pregnancy and Childbirth, World Health Organization, Geneva, Switzerland, 2002.

[28] A. Amjad, U. Amjad, R. Zakar, A. Usman, M. Z. Zakar, and F. Fischer, "Factors associated with caesarean deliveries among child-bearing women in Pakistan: secondary analysis of data from the Demographic and Health Survey, 2012-13," BMC Pregnancy and Childbirth, vol. 18, no. 1, p. 113, 2018.

[29] P. M. Tebeu, E. Mboudou, G. Halle, E. Kongnyuy, E. Nkwabong, and J. N. Fomulu, Risk Factors of Delivery by Caesarean Section in Cameroon (2003-2004): A Regional 
Hospital Report, ISRN Obstetrics and Gynecology, Bethesda, MD, USA, 2011.

[30] B. Gebresilassie, T. Belete, W. Tilahun, B. Berhane, and S. Gebresilassie, "Timing of first antenatal care attendance and associated factors among pregnant women in public health institutions of Axum town, Tigray, Ethiopia, 2017: a mixed design study," BMC Pregnancy and Childbirth, vol. 19, no. 1, p. 340, 2019.

[31] A.-B. Moller, M. Petzold, D. Chou, and L. Say, "Early antenatal care visit: a systematic analysis of regional and global levels and trends of coverage from 1990 to 2013," The Lancet Global Health, vol. 5, no. 10, pp. e977-e983, 2017.

[32] H. Gutema and A. Shimye, "Caesarean section and associated factors at MizanAman general hospital Southwest Ethiopia," Journal of Gynecology and Obstetrics, vol. 2, no. 3, pp. 37-41, 2014.

[33] T. Toan Khanh, B. Eriksson, P. Nhat An, N. Thi Kim Chuc, G. Bondjers, and K. Gottvall, "Technology preference in choices of delivery care utilization from user perspective -A community study in Vietnam," American Journal of Public Health Research, vol. 1, no. 1, pp. 10-17, 2013.

[34] U. Mishra and M. Ramanathan, "Delivery-related complications and determinants of caesarean section rates in India," Health Policy and Planning, vol. 17, no. 1, pp. 90-98, 2002.

[35] A. S. M. Shahabuddin, T. Delvaux, B. Utz, A. Bardají, and V. De Brouwere, "Determinants and trends in health facilitybased deliveries and caesarean sections among married adolescent girls in Bangladesh," BMJ open, vol. 6, no. 9, Article ID e012424, 2016.

[36] R. Mbukani and J. B. S. Z. Kakoma, "Is nulliparity A risk factor for poor obstetrical and neonatal outcomes in Rwandan district hospitals? A prospective observational study AtMuhima district hospital," Rwanda Medical Journal, vol. 69, no. 1, pp. 50-53, 2012. 